319 THE ROLE OF ISAVUCONAZOLE IN TREATMENT OF DISSEMINATED MUCORMYCOSIS IN A 14-YEAR OLD GIRL WITH ACUTE LYMPHOBLASTIC LEUKEMIA

Ernest Bilić, Matej Jelić*, Maja Pavlović, Ana Petrović-Gluščić, Lucija Mucavac, Ivana Mareković, Marija Jandrlić, Daniel Turudić. University Hospital Centre Zagreb

\subsection{6/archdischild-2021-europaediatrics.319}

Invasive mucormycosis is a life-threatening fungal infection in immunocompromised children. It is associated with high mortality rates with limited data concerning the outcome in the pediatric population. Isavuconazole is the newest generation triazole approved for the treatment of invasive mucormycosis in adult patients, but clinical trials have not yet been performed in pediatric population although results of few cases report improvement following the initation of this treatment. We report a case of a 14-year old girl treated for acute lymphoblastic leukemia complicated by destructive necrotic fungal pneumonia and mycotic abscesses on her kidneys. The patient was treated successfully with surgical lobectomy of the inferior lobe of her left lung, nephrectomy and antifungal combination therapy (Amphotericin B and Isavuconazole). At the time of this report, three months after initatiating isavuconazole treatment, the patient continues to receive the drug daily and is doing well. Regarding side effects, we reported transient increase in blood urea and creatinine.

Despite the fact that the Isavuconazole offers new perspectives, further studies are necessary to confirm effectiveness in the pediatric population.

\section{IMMUNE THROMBOCYTOPENIA AND INSTESTINAL PARASITOSIS - IS THERE A CAUSAL LINK?}

${ }^{1}$ Sanela Šalig*, ${ }^{2}$ Izabela Kranjčec, ${ }^{2,3}$ Gordana Jakovljević. 'Varaždin County Health Center, Kolodvorska 20, 42000 Varaždin, Croatia; ${ }^{2}$ Department of Oncology and Hematology, Children's Hospital Zagreb, Klaićeva 16, 10000 Zagreb, Croatia; ${ }^{3}$ School of Medicine, Josip Juraj Strossmayer University of Osijek, Trg Svetog Trojstva 3, 31000 Osijek, Croatia

\subsection{6/archdischild-2021-europaediatrics.320}

Immune thrombocytopenia (ITP) is a relatively common childhood disease, characterized by an isolated decrease in platelet count due to increased degradation by patient's own antibodies, in many cases resulting from a recent viral infection. It is mostly a self-limiting disease in an otherwise healthy child, extremely rarely accompanied by heavy bleeding. The diagnosis is made by excluding secondary causes. The therapeutic strategy from careful monitoring to the first line, which includes immunoglobulins and steroids in various schemes, depends not only on the number of platelets, but on the extent of bleeding, primarily cutaneous and mucosal. Splenectomy as a curative option in the pediatric population occupies only a secondary place, while in cases of chronic disease, newer drugs such as thrombopoietin receptor agonists are resorted to.

A 4.5-year-old boy was hospitalized for severe isolated thrombocytopenia accompanied by hematomas and fever. Laboratory findings verified low platelet counts (Plt 3 x109/L), with normal other bloodlines, inflammatory parameters, and coagulation tests. Cytological analysis of bone marrow punctures confirmed megakaryocyte thrombocytopenia, while antiplatelet antibodies were detected in the patient's serum. As there was no satisfactory increase in platelet counts on initial treatment with intravenous immunoglobulins (approximately $1.5 \mathrm{~g} / \mathrm{kg}$ ), therapy was repeated with pulsed doses of dexamethasone $(24 \mathrm{mg} / \mathrm{m} 2)$, with a mild and temporary effect. Treatment with low doses of prednisone $(2 \mathrm{mg} / \mathrm{kg})$ also did not yield significant results (Plt 20-30 x109/L). Because of the appearance of bloody, diarrheal stools, the treatment was expanded and due to Campylobacter infection, azithromycin therapy was initiated. Ultrasound examination of the abdomen indicated possible intestinal parasitosis, which was confirmed by intestinal passage, while by stool evacuation the macromorphological intestinal parasite was identified as Ascaris lumbricoides. Following mebendazole therapy, there was an immediate and significant increase in platelets (Plt 87 x109/L), which were continuously normal during outpatient follow-up (Plt 320 x109/L).

The association of the etiology of ITP with viral and bacterial infections, certain drugs and vaccines is well known in the literature. The association with parasitosis was based only on anecdotal cases, mainly erythrocyte parasites such as Plasmodium vivax or tissue parasites such as Toxoplasma. Given the resistance of ITP to standard initial therapy and prompt resolution after antiparasitic therapy in our case, a conclusion is imposed about the possible association of ITP with intestinal parasitosis that warrants further research.

\section{THREATMENT OF INFANTILE HEMANGIOMAS WITH PROPRANOLOL}

Andrea Kevrić* , Jelena Roganović. Klinički bolnički centar Rijeka

10.1136/archdischild-2021-europaediatrics.321

Infantile hemangiomas (IH) are benign vascular neoplasms resulting from the proliferation of endothelial vascular cells. They are characterized by unique natural course, with rapid growth in the first year of life (proliferation phase), followed by a slowdown in growth and regression (involution phase) that varies from one to ten years. The aim of the study is to present institutional experiences in the treatment of $\mathrm{IH}$ with propranolol. The study included 37 children with $\mathrm{IH}$, treated at the Division of Hematology, Oncology and Clinical Genetics of the Department of Pediatrics Clinical Hospital Centre Rijeka, in the period from 1 January 2015 to 31 December 2017. Our results have confirmed that IH are more common in females. The majority is localized in the head area. They are mostly solitary lesions. The average diameter was 2,8 ( \pm $1,4) \mathrm{cm}$. The average age at the start of the treatment with propranolol was 3,7 $( \pm 1,1)$ months. The average duration of treatment was $10,1( \pm 5.9)$ months. Propranolol is very effective in the treatment of $\mathrm{IH}$, with complete regression achieved in $70,3 \%$ of children. Side effects are extremely rare (no one recorded in our study), but bear out the initiation of therapy in the hospital setting.

\section{CHRONIC NONBACTERIAL OSTEOMYELITIS IN GIRL TREATED FOR ACUTE LYMPHOBLASTIC LEUKEMIA - CASE REPORT}

Paola Krnjaić*, Izabela Kranjčec, Alenka Gagro, Marijana Šimić Jovićić, Gordana Jakovljević. OB Dubrovnik

10.1136/archdischild-2021-europaediatrics.322 
Introduction Chronic nonbacterial osteomyelitis is a rare autoinflammatory disease of unknown etiology that primarily affects bone and presents with sterile inflammation of the most common metaphyseal areas of the long bones, clavicle, spine, and pelvis. Affected bones are prone to pathologic fractures and slow growth. Laboratory workup results are nonspecific, and skeletal scintigraphy with technetium and magnetic resonance imaging are used as diagnostic imaging modalities. Differentially, malignant events must be considered, such as leukemia and malignant bone tumors, chronic infectious osteomyelitis, avascular necrosis, etc. If the diagnosis is unclear, a bone biopsy with bacteriological analysis of the bone sample is required to exclude infectious osteomyelitis.

Case Report An 8-year-old girl was treated for acute lymphoblastic leukemia of $\mathrm{T}$ immunophenotype, high risk, according to ALL IC-BFM 2009 protocol, early complete remission was achieved. Intensive chemotherapy treatment was characterized by a number of complications, including frequent febrile neutropenia, invasive fungal lung disease, and acute neurotoxicity in the form of cerebral ischemia. After completion of reinduction therapy, a period of persistent bone marrow aplasia followed by recurrent and prolonged sepsis caused by a highly resistant strain of Pseudomonas aeruginosa of unknown origin preceded maintenance therapy. Because the girl complained of foot pain with inability to walk during maintenance therapy, extensive diagnostic workup was performed, including technetium bone scintigraphy and magnetic resonance imaging, to verify an osteomyelitis focus in the left calcaneus with intense bone remodeling of SI and shoulder joint. After completion of treatment for sepsis in the control laboratory findings continued slightly elevated C-reactive protein with normal sedimentation of erythrocytes and procalcitonin. Pathohistological analysis of bone biopsy showed chronic inflammatory reaction, while microbiological analysis was negative. Skin lesions were not observed, HLA typing was negative, anamnestic data of maternal inflammatory bowel disease was known. In collaboration with an immunoreumatologist and an orthopedist, chronic nonbacterial osteomyelitis was treated and treatment with bisphosphonates was started. according to a standard regimen, with an excellent clinical response. The girl was pain free six months after starting treatment and physical therapy. The underlying malignancy is still in remission, inflammatory parameters are normal, while control imaging still continues.

Conclusion Chronic nonbacterial osteomyelitis should be considered as a differential diagnosis in chronic inflammatory bone lesions without an isolated microbiologic agent and in patients with acute lymphocytic leukemia. Therapeutic options include nonsteroidal anti-inflammatory drugs, disease-modifying antirheumatic drugs (DMARDs), corticosteroids, and bisphosphonates. However, given the possibility of influencing hematologic findings, bisphosphonate therapy is the treatment of choice if oncologic disease requires further treatment.

\section{TREATMENT RESULTS OF T-LYMPHOBLASTIC LYMPHOMA IN CHILDREN - A SINGLE-CENTER EXPERIENCE}

\footnotetext{
${ }^{1,2}$ Ernest Bilić, ${ }^{2}$ Petra Ivančićc, ${ }^{1}$ Maja Pavlović, ${ }^{1}$ Ranka Femenić, ${ }^{1}$ Ana Petrović-Gluščić,

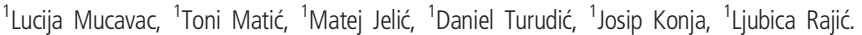
${ }^{1}$ Department of Pediatrics, Division of Hematology and Oncology, University Hospital Centre Zagreb, Zagreb, Croatia; ${ }^{2}$ School of Medicine, University of Zagreb, Zagreb, Croatia
}

10.1136/archdischild-2021-europaediatrics.323
Aim Our study aimed to determine the overall survival of children with non-Hodgkin T-lymphoblastic lymphoma (TLBL), analyze and present patient characteristics and initial presentation of their disease.

Methods Our study included all patients with T-LBL nonHodgkin lymphoma treated in the Department of Pediatrics, Division of Hematology and Oncology, University Hospital Centre Zagreb between January 1st, 2002 and December 31st, 2017. Relevant information including general patient data (gender, age at the time of diagnosis), initial clinical presentation (the presence of mediastinal masses, pericardial and pleural effusion, the presence of bone marrow and central nervous system disease) and treatment information (used therapeutic protocol and mortality data) was collected from the available medical documentation. The Kaplan-Meier curve shows patients' survival.

Results Overall, 19 patients were included, 14 of which were male $(73.68 \%)$ and 5 were female $(26.32 \%)$. The median age at diagnosis was 10 years. 17 patients (94.44\%) presented with mediastinal mass and 9 of them $(52.94 \%)$ had pleural effusion. At the time of diagnosis, pericardial effusion and superior vena cava syndrome were present in $6(33.33 \%)$ and $3(16.67 \%)$ patients, respectively. Bone marrow involvement was detected in 10 patients (52.63\%), whereas CNS involvement in only one (5.26\%). 16 out of 19 patients survived (84.21\%; 95\% CI 67.81-100). Survival was higher for boys $(85.71 \%)$ than for girls $(80 \%)$, but the difference was not statistically significant $(\mathrm{p}=0.764)$.

Conclusion Comparing general epidemiologic data with the one in the available literature we haven't found a significant deviation of the prevalence of the disease between the sex or the median age of onset of the disease. The result of $90 \%$ five-year survival of children with T-LBL in the BFM group study was not repeated in later studies and the overall survival of $84 \%$ in our group was consistent with the results reported in the available literature.

\section{NEUTROPENIA IN CHILDREN}

${ }^{1,2}{ }^{2}$ rnest Bilićx, ${ }^{2}$ Kristijan Lujić, ${ }^{1}$ Maja Pavlović, 'Lucija Mucavac, ${ }^{1} T o n i$ Matić, ${ }^{1}$ Daniel Turudić, ${ }^{1}$ Matej Jelić, ${ }^{2}$ Hana Chudy. ${ }^{1}$ University Hospital Centre Zagreb, Department of Pediatrics, Division of Pediatric Hematology and Oncology; ${ }^{2}$ School of Medicine, University of Zagreb, Zagreb, Croatia

\subsection{6/archdischild-2021-europaediatrics.324}

Aim The aim of this study was to show presentation of individual types of neutropenia as well as their outcome depending on the lowest absolute neutrophil count (ANC), period in which ANC was below 1000 and presence of infections during the duration of neutropenia.

Materials and Methods In this study 288 children with neutropenia were observed in the period from 2005-2020. The study was provided at the Division of Pediatric Hematology and Oncology at the University Hospital Centre Zagreb, Croatia.

Results $139(48,26 \%)$ examinees were female and 149 $(51,74 \%)$ were male. Of all the examinees 265 (92,01\%) were diagnosed before their seventh birthday while 175 (60,76\%) had diagnosis made during their first year of life. Retrospective analysis revealed that $281(97,57 \%)$ examinees had acquired neutropenia and only $7(2,51 \%)$ had some type of congenital neutropenia ( 3 had severe congenital neutropenia, 2 had cyclic neutropenia, 1 had Shwachman-Diamond syndrome 